Ergod. Th. \& Dynam. Sys. (2222), 11, 11

Printed in the United Kingdom

(c) 2222 Cambridge University Press

\title{
A Family of Piecewise Expanding Maps having Singular Measure as a limit of ACIM's
}

\author{
Zhenyang Li†,Paweł Góra†, Abraham Boyarsky†, \\ Harald Proppe† and Peyman Eslami† \\ $\dagger \dagger$ Department of Mathematics and Statistics, Concordia University, 1455 de \\ Maisonneuve Blvd. West, Montreal, Quebec H3G 1M8, Canada \\ (e-mail: zhenyangemail@gmail.com),(e-mail: pgora@mathstat.concordia.ca), \\ (e-mail: boyar@alcor.concordia.ca),(e-mail: proppe@alcor.concordia.ca), \\ (e-mail: peyman_eslami@yahoo.com)
}

(Received September 20, 2011)

\begin{abstract}
Keller [9] introduced families of W-shaped maps that can have a great variety of behavior. As a family approaches a limit $\mathrm{W}$ map, he observed behavior that was either described by a probabilty density function (pdf) or by a singular point measure. Based on this, Keller conjectured that instability of the absolutely continuous invariant measure (acim) can result only from the existence of small invariant neighbourhoods of the fixed critical point of the limit map. In this note we show that the conjecture is not true. We construct a very simple family of W maps with acim's supported on the whole interval, whose limiting dynamical behavior is captured by a singular measure. Key to the analysis is the use of a general formula for invariant densities of piecewise linear and expanding maps [6].
\end{abstract}

\section{Introduction}

Usually, the absolutely continuous invariant measure of a piecewise expanding map of an interval is stable under deterministic or even random perturbations. This means that if we consider a family of piecewise expanding maps $\tau_{a}, a>0$ with acims $\mu_{a}$, converging to a piecewise expanding map $\tau_{0}$ with acim $\mu_{0}$, then under general assumptions $\mu_{a}$ s converge to $\mu_{0}$. One such assumption is that for some positive $\varepsilon,\left|\tau_{a}^{\prime}\right|>2+\varepsilon$ for all $a \geq 0$. Actually, much more is proven in [10] and [1]. This stability property is often useful, for example, in establishing results about metastable systems [14].

$\dagger$ The research of the authors was supported by NSERC grants.

Prepared using etds.cls [Version: 1999/07/21 v1.0] 
Keller [9] introduced the family of $W$-maps that are piecewise expanding and exhibit a wide variety of behaviour. This was done to understand whether in dimension one the expanding constant ensuring stability is really 2 rather than 1 as for zero-dimensional systems [7]. This regularity was later confirmed in [8] by showing that this constant for a piecewise expanding $n$-dimensional system with rectangular partition is $n+1$.

Key to the complexity of Keller's families is the fact that, as the parameter approaches 0, say, the behavior near a folded critical point plays a crucial role. This critical point has slope 2 on one side and -2 on the other. Thus, the entire family is uniformly piecewise expanding and each member has a unique absolutely continuous invariant measure. However, the stability of probability density functions that one might expect in families of uniformly piecewise expanding maps does not occur. Keller provided an example of a family for which the limit of acims is a singular measure. This occurred because of the existence of diminishing invariant neighbourhoods of the critical point. Keller conjectured that this is the only mechanism which can cause such limiting behaviour.

In this paper we construct a family of simple $W$-maps which disproves Keller's conjecture. All our maps are piecewise expanding with slopes strictly greater than 2 in magnitude and are exact with their acim's supported on all of $[0,1]$.

Standard bounded variation methods cannot be applied in this setting as the slopes of maps in our family are not uniformly bounded away from 2 . The LasotaYorke BV inequality [2] cannot be invoked for families of maps whose slopes are not bounded away from 2 in magnitude. Other research related to the objectives of this note can be found in [3], [13] and [11]. However, none of the methods used in these papers apply to the family of maps we consider.

In a recent paper [5], a 3-parameter countable family of transitive maps was constructed which converges to the $W$-map for which the acim's approach any given convex combination of the point measure at $1 / 2$ and the $W$-map acim. All maps in this family are Markov, which makes the analysis easier.

In this note we shall utilize the main result of $[\mathbf{6}]$ which proves that the invariant pdf for any piecewise linear map which is eventually expanding has a convenient infinite series expansion. The estimates on the family of pdfs derived from this representation allows us to prove our main result, that the acim's of the family of $W$-maps approach a combination of an absolutely continuous and a singular measure rather than the acim of the limit map.

In Section 2 we introduce our family and state the main theorem, which is proved in Section 3. In Section 4, we show computational results for some pdfs of the $W_{a}$ maps when $a$ is small.

Prepared using etds.cls 
2. Family of $W_{a}$ maps and the main result

We consider the family $\left\{W_{a}: 0 \leq a\right\}$ of maps of $[0,1]$ onto itself defined by

$$
W_{a}(x)=\left\{\begin{array}{l}
1-4 x, \text { for } 0 \leq x<1 / 4 ; \\
(2+a)(x-1 / 4), \text { for } 1 / 4 \leq x<1 / 2 \\
1 / 2+a / 4-(2+a)(x-1 / 2), \text { for } 1 / 2 \leq x<3 / 4 \\
4(x-3 / 4), \text { for } 3 / 4 \leq x \leq 1
\end{array}\right.
$$

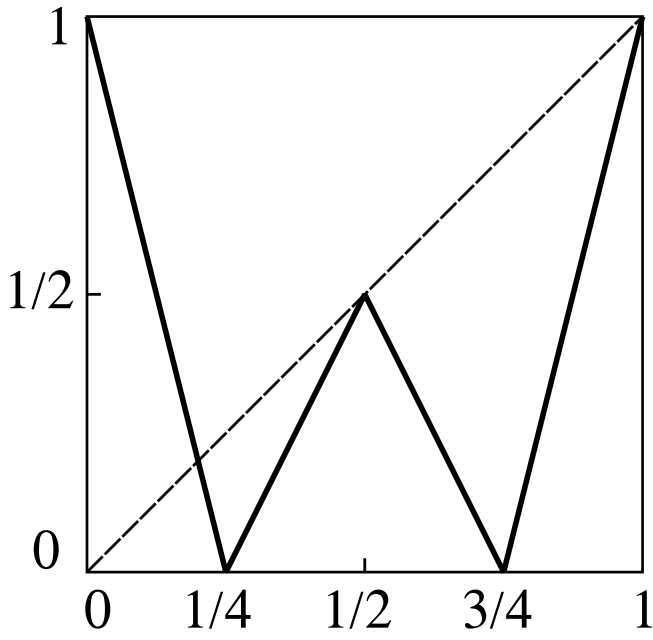

a)

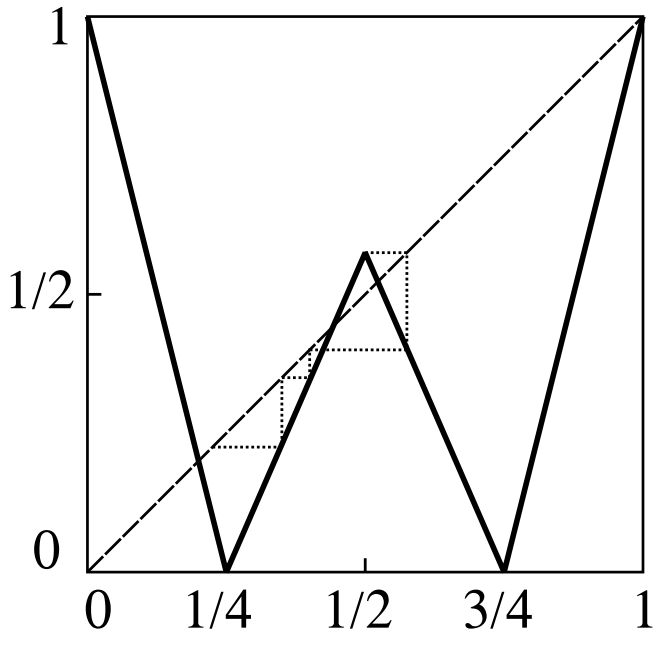

b)

FiguRE 1. a) map $W_{0}$, b) map $W_{a}, a>0$, with a few first points of the trajectory of $1 / 2$.

The map $W_{0}$ is Keller's $W$-map [9]. We consider only small $a>0$ as we are interested in the limiting behaviour of the $W_{a}$ 's as $a \rightarrow 0$. Fig. 1 shows the graphs of $W_{a}$ for $a=0$ and $a>0$. Every $W_{a}$ is a piecewise linear, piecewise expanding map with minimal modulus of the slope equal to $2+a$. Every $W_{a}$ has a unique acim $\mu_{a}$ supported on $[0,1]$ and is exact with respect to this measure. The transitivity of such maps is proven in [5], and the uniqueness of acim and exactness follow directly from the Li-Yorke paper [12].

Let $h_{a}$ denote the normalized density of $\mu_{a}, a \geq 0$. It is easy to check that for $W_{0}, \mu_{0}$ has density

$$
h_{0}= \begin{cases}\frac{3}{2}, & \text { for } 0 \leq x<1 / 2 \\ \frac{1}{2}, & \text { for } 1 / 2 \leq x \leq 1\end{cases}
$$

Our goal is to prove the following theorem:

THEOREM 2.1. As $a \rightarrow 0$ the measures $\mu_{a}$ converge $*$-weakly to the measure

$$
\frac{2}{3} \mu_{0}+\frac{1}{3} \delta_{\left(\frac{1}{2}\right)}
$$

where $\delta_{\left(\frac{1}{2}\right)}$ is the Dirac measure at point $1 / 2$.

Prepared using etds.cls 
The proof relies on the general formula for invariant densities of piecewise linear maps [6] and direct calculations. The calculations depend on the parameter $a$, but we suppress it whenever there is no confusion.

\section{Proofs}

This section contains the proof of Theorem 2.1, divided into a number of steps.

3.1. Formula for non-normalized invariant density of $W_{a}$ We adapt the general formulas of $[\mathbf{6}]$ to our case and obtain the following formula for $f_{a}$ :

Lemma 3.1. For small $a>0$ there exists $A<-1$ such that

$$
f_{a}=1+2 A\left(\sum_{n=1}^{\infty} \frac{\chi^{s}\left(\beta(1 / 2, n), W_{a}^{n}(1 / 2)\right)}{|\beta(1 / 2, n)|}\right) .
$$

is a $W_{a}$-invariant non-normalized density.

Here,

$$
\chi^{s}(t, y)= \begin{cases}\chi_{[0, y]} & \text { for } t>0 \\ \chi_{[y, 1]} & \text { for } t<0\end{cases}
$$

and $\beta(1 / 2, n)$ is the cumulative slope along the $n$ steps of the trajectory of $1 / 2$ defined by:

$$
\beta(1 / 2,1)=2+a, \text { and }
$$

$\beta(1 / 2, n)=(2+a) \cdot W_{a}^{\prime}\left(W_{a}(1 / 2)\right) \cdot W_{a}^{\prime}\left(W_{a}^{2}(1 / 2)\right) \cdots W_{a}^{\prime}\left(W_{a}^{n-1}(1 / 2)\right)$, for $n \geq 2$.

The detailed justification of formula (3) is in Subsection 3.2.

For small positive $a$, the first image of $1 / 2$ is $W_{a}(1 / 2)=1 / 2+a / 4$ and the next image lands just below the fixed point slightly less than $1 / 2$. The following forward images of $1 / 2$ form a decreasing sequence until they go below $1 / 4$. Let $k$ be the first iterate $j$ when $W_{a}^{j}(1 / 2)$ is less than $1 / 4$. That is, $k=\min \left\{j \geq 1: W_{a}^{j}(1 / 2) \leq 1 / 4\right\}$. Then, the consecutive cumulative slopes of $1 / 2$, namely $\beta(1 / 2, j), 1 \leq j \leq k$, are

$$
(2+a),-(2+a)^{2},-(2+a)^{3}, \ldots,-(2+a)^{k},
$$

and

$$
f_{a}=1+2 A\left(\frac{\chi_{\left[0, W_{a}(1 / 2)\right]}}{(2+a)}+\sum_{j=2}^{k} \frac{\chi_{\left[W_{a}^{j}(1 / 2), 1\right]}}{(2+a)^{j}}+\ldots\right)
$$

3.2. Justification of the formula for $f_{a}$ Using the notation of [6], we have the following lemma:

LEMma 3.2. (a) $N=4, K=2, L=0$;

(b) $\alpha=(1,1 / 2+a / 4,1 / 2+a / 4,1), \beta=(-4,2+a,-(2+a), 4), \gamma=(0,0,0,0)$;

(c) The digits $A=\left(a_{1}, a_{2}, a_{3}, a_{4}\right)$, where $a_{1}=-1, a_{2}=1 / 2+a / 4, a_{3}=$ $-3 / 2-3 a / 4, a_{4}=3$;

Prepared using etds.cls 
(d) There are two $c_{i}$ 's, which are $c_{1}=(1 / 2,2)$ and $c_{2}=(1 / 2,3)$, and $j\left(c_{1}\right)=2$, $j\left(c_{2}\right)=3$. Then, $W_{u}=\left\{c_{1}, c_{2}\right\}, W_{l}=\emptyset, U_{l}=\left\{c_{2}\right\}, U_{r}=\left\{c_{1}\right\}$.

(e) $\beta\left(c_{1}, 1\right)=2+a$ since $j\left(c_{1}\right)=2$, then $\beta\left(c_{1}, 2\right)=-(2+a)^{2}$ and $\beta\left(c_{1}, k\right)=$ $-(2+a)^{k}$ up to $k$ defined in Subsection 3.1, $k=\min \left\{j \geq 1: W_{a}^{j}(1 / 2) \leq 1 / 4\right\}$;

(f) $\beta\left(c_{2}, 1\right)=-(2+a)$ since $j\left(c_{2}\right)=3$, then $\beta\left(c_{2}, 2\right)=(2+a)^{2}$ and $\beta\left(c_{2}, k\right)=(2+a)^{k}$ up to the same $k$ in part $(e), W_{a}^{n}\left(c_{1}\right)=W_{a}^{n}\left(c_{2}\right)$ for all $n$;

(g) Based on $(f)$, we have the following for the matrix $S=S_{i, j}, i, j=1,2$ :

For $c_{1} \in U_{r}$

$$
\begin{aligned}
S_{1,1} & =\sum_{n=1}^{\infty} \frac{\delta\left(\beta\left(\left(c_{1}, n\right)>0\right)\right) \delta\left(W_{a}^{n}\left(c_{1}\right)>1 / 2\right)+\delta\left(\beta\left(\left(c_{1}, n\right)<0\right)\right) \delta\left(W_{a}^{n}\left(c_{1}\right)<1 / 2\right)}{\left|\beta\left(c_{1}, n\right)\right|} \\
S_{1,2} & =\sum_{n=1}^{\infty} \frac{\delta\left(\beta\left(\left(c_{1}, n\right)>0\right)\right) \delta\left(W_{a}^{n}\left(c_{1}\right)>1 / 2\right)+\delta\left(\beta\left(\left(c_{1}, n\right)<0\right)\right) \delta\left(W_{a}^{n}\left(c_{1}\right)<1 / 2\right)}{\left|\beta\left(c_{1}, n\right)\right|} .
\end{aligned}
$$

For $c_{2} \in U_{l}$

$$
\begin{aligned}
S_{2,1} & =\sum_{n=1}^{\infty} \frac{\delta\left(\beta\left(\left(c_{2}, n\right)<0\right)\right) \delta\left(W_{a}^{n}\left(c_{2}\right)>1 / 2\right)+\delta\left(\beta\left(\left(c_{2}, n\right)>0\right)\right) \delta\left(W_{a}^{n}\left(c_{2}\right)<1 / 2\right)}{\left|\beta\left(c_{2}, n\right)\right|}, \\
S_{2,2} & =\sum_{n=1}^{\infty} \frac{\delta\left(\beta\left(\left(c_{2}, n\right)<0\right)\right) \delta\left(W_{a}^{n}\left(c_{2}\right)>1 / 2\right)+\delta\left(\beta\left(\left(c_{2}, n\right)>0\right)\right) \delta\left(W_{a}^{n}\left(c_{2}\right)<1 / 2\right)}{\left|\beta\left(c_{2}, n\right)\right|},
\end{aligned}
$$

where $\delta$ ("condition") is equal to 1 if the "condition" holds and to 0 if it does not.

Remark: It follows from $(e, f)$ of Lemma 3.2 that $S_{i, j}$ are equal for $i, j=1,2$. Let $I d$ be the $2 \times 2$ identity matrix, $V=[1,1]$. Then, for the solution, $D=\left[D_{1}, D_{2}\right]$, of the following system :

$$
\left(-S^{T}+I d\right) D^{T}=V^{T}
$$

we have $D_{1}=D_{2}$. Let us denote them by $\mathrm{A}$.

Let $I_{1}, I_{2}, I_{3}, I_{4}$ be the partition of $I=[0,1]$, where $I_{1}=[0,1 / 4), I_{2}=$ $(1 / 4,1 / 2), I_{3}=(1 / 2,3 / 4)$ and $I_{4}=(3 / 4,1]$. Let $\beta_{1}=-4, \beta_{2}=2+a, \beta_{3}=-(2+a)$, and $\beta_{4}=4$. We define the following index:

$$
j(x)=j \text { for } x \in I_{j}, j=1,2,3,4,
$$

and

$$
j\left(c_{1}\right)=2, j\left(c_{2}\right)=3 .
$$

Already defined for Lemma 3.1 we have cumulative slopes for iterates of points:

$$
\beta(x, 1)=\beta_{j(x)}, \quad \text { and } \beta(x, n)=\beta(x, n-1) \cdot \beta_{j\left(W_{a}^{n-1}(x)\right)}, \quad n \geq 2,
$$

and

$$
\chi^{s}(t, y)= \begin{cases}\chi_{[0, y]} & \text { for } t>0 \\ \chi_{[y, 1]} & \text { for } t<0\end{cases}
$$

Using Theorem 2 in [6] directly, we obtain Lemma 3.2. Now, we can prove Lemma 3.1:

Prepared using etds.cls 
Proof: First, by part ( $g$ ) of Lemma 3.2, since the first and fourth branches of $W_{a}$ have slope of modulus $4>2+a$,

$$
S_{i, j} \leq \sum_{n=1}^{\infty} \frac{1}{(2+a)^{n}}=\frac{1}{1+a}<1 .
$$

On the other hand, for small $a$

$$
S_{i, j} \geq \frac{1}{2+a}+\frac{1}{(2+a)^{2}}>1 / 2 .
$$

Now, the solution of the system (5) will be $D_{1}=D_{2}=\frac{1}{1-2 S_{1,1}}<-1$. By Theorem 2 in $[6]$, it follows from $(d, e, f)$ of Lemma 3.2 that:

$$
\begin{aligned}
f_{a} & =1+D_{1} \sum_{n=1}^{\infty} \frac{\chi^{s}\left(\beta\left(c_{1}, n\right), W_{a}^{n}\left(c_{1}\right)\right)}{\left|\beta\left(c_{1}, n\right)\right|}+D_{2} \sum_{n=1}^{\infty} \frac{\chi^{s}\left(-\beta\left(c_{2}, n\right), W_{a}^{n}\left(c_{2}\right)\right)}{\left|\beta\left(c_{2}, n\right)\right|} \\
& =1+A \sum_{n=1}^{\infty} \frac{\chi^{s}\left(\beta\left(c_{1}, n\right), W_{a}^{n}(1 / 2)\right)}{\left|\beta\left(c_{1}, n\right)\right|}+A \sum_{n=1}^{\infty} \frac{\chi^{s}\left(-\beta\left(c_{2}, n\right), W_{a}^{n}(1 / 2)\right)}{\left|\beta\left(c_{2}, n\right)\right|} \\
& =1+2 A\left(\sum_{n=1}^{\infty} \frac{\chi^{s}\left(\beta(1 / 2, n), W_{a}^{n}(1 / 2)\right)}{|\beta(1 / 2, n)|}\right),
\end{aligned}
$$

which completes the proof.

3.3. Estimates on $f_{a}$ Recall that $k=\min \left\{j \geq 1: W_{a}^{j}(1 / 2) \leq 1 / 4\right\}$. Clearly, $k>1$. Furthermore, we have the following lemma:

LEMMA 3.3. (I) for $2 \leq m \leq k, W_{a}^{m}(1 / 2)=-\frac{1}{4} a \frac{a(2+a)^{m-1}+1}{1+a}+\frac{1}{2}$;

(II) $\lim _{a \rightarrow 0} a k=0$;

$(I I I) \lim _{a \rightarrow 0} \frac{1}{a(2+a)^{k}}=0$.

Moreover, if we let $k_{1}=\left[\frac{2}{3} k\right]$ (integer part of $2 k / 3$ ), we have

$(I V) \lim _{a \rightarrow 0} \frac{1}{a(2+a)^{k_{1}}}=0$;

$(V) \lim _{a \rightarrow 0} a^{2}(2+a)^{k_{1}}=0$

$(V I) \lim _{a \rightarrow 0} W_{a}^{k_{1}}\left(\frac{1}{2}\right)=\frac{1}{2}$.

Proof: Suppose (I) is true. By the definition of $k, 0 \leq W_{a}^{k-1}(1 / 2) \leq 1 / 4$. That is,

$$
0 \leq-\frac{1}{4} a \frac{a(2+a)^{k-1}+1}{1+a}+\frac{1}{2} \leq \frac{1}{4} .
$$

The first inequality of (6) implies

$$
a^{2}(2+a)^{k-2} \leq 1
$$

and the second implies

$$
a^{2}(2+a)^{k-1} \geq 1
$$

and so

$$
2 \leq \liminf _{a \rightarrow 0} a^{2}(2+a)^{k} \leq \limsup _{a \rightarrow 0} a^{2}(2+a)^{k} \leq 4
$$

Prepared using etds.cls 
Assertions (II), (III) and (V) follow immediately. To prove (IV) we have from (7)

$$
a \leq \frac{2+a}{(2+a)^{k / 2}}
$$

and so using also (8):

$$
\begin{aligned}
\frac{1}{a(2+a)^{k_{1}}} & =\frac{(2+a)^{k-k_{1}}}{a(2+a)^{k}} \leq \frac{a(2+a)^{k-k_{1}}}{2+a} \\
& \leq \frac{2+a}{(2+a)^{k / 2}} \frac{1}{2+a}(2+a)^{k-k_{1}}=\frac{1}{(2+a)^{k_{1}-k / 2}} \rightarrow 0
\end{aligned}
$$

Finally (VI) follows from (I) and (V).

Now, let us prove (I). For $m=2$, it is easy to check that $W_{a}^{2}(1 / 2)=\frac{2-a-a^{2}}{4}$ which is the same as $-\frac{1}{4} a \frac{a(2+a)+1}{1+a}+\frac{1}{2}$. Suppose (I) holds for $m=i<k$, that is

$$
W_{a}^{i}(1 / 2)=-\frac{1}{4} a \frac{a(2+a)^{i-1}+1}{1+a}+\frac{1}{2} .
$$

Then for $m=i+1$,

$$
\begin{aligned}
W_{a}^{i+1}(1 / 2) & =(2+a)\left(-\frac{1}{4} a \frac{a(2+a)^{i-1}+1}{1+a}+\frac{1}{2}-\frac{1}{4}\right) \\
& =-\frac{1}{4} a \frac{a(2+a)^{i}+2+a}{1+a}+\frac{1}{2}+\frac{a}{4} \\
& =-\frac{1}{4} a \frac{a(2+a)^{i}+1}{1+a}+\frac{1}{2}
\end{aligned}
$$

This completes the proof.

Let $\delta$ ("condition") be equal to 1 if the "condition" holds and to 0 if it does not. Lemma 3.2 implies that

$S_{1,1}=\sum_{n=1}^{\infty} \frac{\delta(\beta((1 / 2, n)>0)) \delta\left(W_{a}^{n}(1 / 2)>1 / 2\right)+\delta(\beta((1 / 2, n)<0)) \delta\left(W_{a}^{n}(1 / 2)<1 / 2\right)}{|\beta(1 / 2, n)|}$.

Also, it was shown there that $A=\frac{1}{1-2 S_{1,1}}$. Since

$$
S_{1,1} \geq \sum_{n=1}^{k_{1}} \frac{1}{(2+a)^{n}}=\frac{\frac{1}{2+a}-\frac{1}{(2+a)^{k_{1}+1}}}{1-\frac{1}{2+a}}
$$

and

$$
S_{1,1} \leq \sum_{n=1}^{\infty} \frac{1}{(2+a)^{n}}=\frac{1}{1+a}
$$

we have

$$
A_{l}=\frac{1+a}{a-1+\frac{2}{(2+a)^{k_{1}}}} \leq A \leq \frac{1+a}{a-1}=A_{h} .
$$

Note that, for small $a$, both estimates $A_{l}$ and $A_{h}$ are smaller that -1 .

Prepared using etds.cls 
Let us define,

$$
g_{l}=\frac{\chi_{\left[0, W_{a}(1 / 2)\right]}}{(2+a)}+\sum_{j=2}^{k_{1}} \frac{\chi_{\left[W_{a}^{j}(1 / 2), 1\right]}}{(2+a)^{j}}
$$

and

$$
g_{h}=g_{l}+\sum_{j=k_{1}+1}^{\infty} \frac{1}{(2+a)^{j}}=g_{l}+\frac{1}{(1+a)(2+a)^{k_{1}}} .
$$

Let us further define $f_{l}=1+2 A_{l} g_{h}$ and $f_{h}=1+2 A_{h} g_{l}$. It follows from (4) and (9) that

$$
f_{l} \leq f_{a} \leq f_{h} .
$$

Let $\chi_{1}=\chi_{[0,1 / 2+a / 4]}, \chi_{j}=\chi_{\left[W_{a}^{j}(1 / 2), 1 / 2+a / 4\right]}, j=2,3, \ldots, k_{1}, \chi_{c}=\chi_{(1 / 2+a / 4,1]}$. Now we will represent the functions $f_{l}$ and $f_{h}$ as combinations of functions $\chi_{j}$, $j=1, \ldots, k_{1}$ and $\chi_{c}$. After some calculations, we obtain:

$$
\begin{aligned}
f_{l}= & \left(\frac{2}{2+a} A_{l}+1\right) \chi_{1}+2 A_{l} \sum_{n=2}^{k_{1}} \frac{\chi_{n}}{(2+a)^{n}}+ \\
& +\left(2 A_{l} \frac{\frac{1}{2+a}-\frac{1}{(2+a)^{k_{1}}}}{1+a}+1\right) \chi_{c}+2 A_{l} \frac{1}{(1+a)(2+a)^{k_{1}}} \\
f_{h}= & {\left[A_{h} \frac{2}{2+a}+1\right] \chi_{1}+2 A_{h} \sum_{n=2}^{k_{1}} \frac{\chi_{n}}{(2+a)^{n}}+\left(2 \frac{\frac{1}{2+a}-\frac{1}{(2+a)^{k_{1}}}}{a-1}+1\right) \chi_{c} . }
\end{aligned}
$$

Note that (9) implies that both $A_{l}, A_{h}$ are smaller than $-(1+2 a)$. Using this we can show that all coefficients in the representation of $f_{l}$ and $f_{h}$ are negative for sufficiently small $a$.

3.4. Normalization Let us define $J_{1}=\left[0, W_{a}^{k_{1}}(1 / 2)\right], J_{2}=\left(W_{a}^{k_{1}}(1 / 2), 1 / 2+a / 4\right]$, $J_{3}=(1 / 2+a / 4,1]$. We will calculate integrals of $f_{h}$ over each of these intervals and use them to normalize $f_{h}$. We have

$$
\begin{aligned}
C_{1}=\int_{J_{1}} f_{h} d \lambda & =\int_{J_{1}}\left[2\left(\frac{1+a}{a-1} \frac{1}{2+a}\right)+1\right] \chi_{1} d \lambda \\
& =\left[2\left(\frac{1+a}{a-1} \frac{1}{2+a}\right)+1\right] W_{a}^{k_{1}}\left(\frac{1}{2}\right)=\frac{a^{2}+3 a}{(a-1)(2+a)} W_{a}^{k_{1}}\left(\frac{1}{2}\right) .
\end{aligned}
$$

Using Lemma 3.3, we have $\lim _{a \rightarrow 0} \frac{C_{1}}{a}=-\frac{3}{4}$. In the same way we can see that for any $0<\alpha<1 / 2$, we obtain

$$
\lim _{a \rightarrow 0} \frac{1}{a} \int_{0}^{\alpha} f_{h} d \lambda=-\frac{3}{2} \alpha
$$

Prepared using etds.cls 
On the interval $J_{2}$, the integral of $f_{h}$ is:

$$
\begin{aligned}
C_{2}=\int_{J_{2}} f_{h} d \lambda= & \int_{J_{2}}\left[2\left(\frac{1+a}{a-1} \frac{1}{2+a}\right)+1\right] \chi_{1} d \lambda+2 \frac{1+a}{a-1} \sum_{j=2}^{k_{1}} \int_{J_{2}} \frac{\chi_{j}}{(2+a)^{j}} d \lambda \\
= & \frac{a^{2}+3 a}{(a-1)(2+a)}\left(\frac{1}{2}+\frac{a}{4}-W_{a}^{k_{1}}\left(\frac{1}{2}\right)\right) \\
& +2 \frac{1+a}{a-1}\left[\frac{\left(k_{1}-1\right) a^{2}}{4(2+a)(1+a)}+\frac{a}{4(1+a)} \frac{1-\frac{1}{(2+a)^{k_{1}-1}}}{1+a}\right] .
\end{aligned}
$$

Using Lemma 3.3, we have

$$
\lim _{a \rightarrow 0} \frac{C_{2}}{a}=-\frac{1}{2} .
$$

On the interval $J_{3}$, the integral of $f_{h}$ is:

$$
\begin{aligned}
C_{3}=\int_{J_{3}} f_{h} d \lambda & =\int_{J_{3}}\left(2 \frac{\frac{1}{2+a}-\frac{1}{(2+a)^{k_{1}}}}{a-1}+1\right) \chi_{c} d \lambda \\
& =\left(2 \frac{\frac{1}{2+a}-\frac{1}{(2+a)^{k_{1}}}}{a-1}+1\right)\left(\frac{1}{2}-\frac{a}{4}\right) .
\end{aligned}
$$

Using Lemma 3.3, we have

$$
\lim _{a \rightarrow 0} \frac{C_{3}}{a}=-\frac{1}{4} .
$$

In the same way we can see that for any $0<\alpha<1 / 2$, we obtain

$$
\lim _{a \rightarrow 0} \frac{1}{a} \int_{1 / 2+\alpha}^{1} f_{h} d \lambda=-\frac{1}{2}\left(\frac{1}{2}-\alpha\right) .
$$

If we define $B=C_{1}+C_{2}+C_{3}$, then $\frac{f_{h}}{B}$ is a normalized density. We see that

$$
\lim _{a \rightarrow 0} \frac{B}{a}=-\frac{3}{2} \text {. }
$$

3.5. Conclusion of the proof Now, we will use our foregoing calculations to show that the normalized measures $\left(f_{h} / B\right) \cdot \lambda$ converge $*$-weakly to the measure $\frac{2}{3} \mu_{0}+\frac{1}{3} \delta_{\left(\frac{1}{2}\right)}$, as $a \rightarrow 0$.

For any interval $[0, \alpha], 0<\alpha<1 / 2$ as $a \rightarrow 0$, formula (11) implies

$$
\lim _{a \rightarrow 0} \int_{0}^{\alpha} \frac{f_{h}}{B} d \lambda=\frac{-\frac{3}{2} \alpha}{-\frac{3}{2}}=\alpha .
$$

For $J_{2}$, which converges to the point $1 / 2$, formula (12) implies

$$
\lim _{a \rightarrow 0} \int_{J_{2}} \frac{f_{h}}{B} d \lambda=\frac{-\frac{1}{2}}{-\frac{3}{2}}=\frac{1}{3} .
$$

For any interval $[1 / 2+\alpha, 1], 0<\alpha<1 / 2$, formula (13) implies

$$
\lim _{a \rightarrow 0} \int_{1 / 2+\alpha}^{1} \frac{f_{h}}{B} d \lambda=\frac{-\frac{1}{2}\left(\frac{1}{2}-\alpha\right)}{-\frac{3}{2}}=\frac{1}{3}\left(\frac{1}{2}-\alpha\right) \text {. }
$$

Prepared using etds.cls 
Formulas $(14),(15)$ and (16) together show that measures $\left(f_{h} / B\right) \cdot \lambda$ converge $*-$ weakly to the sum of the measure with density $\chi_{[0,1 / 2]}+\frac{1}{3} \chi_{[1 / 2,1]}$ and $\frac{1}{3}$ of a unit point mass at $1 / 2$, i.e., to the measure $\frac{2}{3} \mu_{0}+\frac{1}{3} \delta_{\left(\frac{1}{2}\right)}$.

Now, we will show the same for the normalized measure defined using $f_{l}$. To this end, let us note that

$$
\begin{aligned}
f_{h}-f_{l} & =2 A_{h} g_{l}-2 A_{l} g_{h}=2\left(A_{h}-A_{l}\right) g_{l}-2 A_{l} \frac{1}{(1+a)(2+a)^{k_{1}}} \\
& =2 \frac{1+a}{a-1} \frac{-2 /(2+a)^{k_{1}}}{a-1+2 /(2+a)^{k_{1}}} g_{l}-2 A_{l} \frac{1}{(1+a)(2+a)^{k_{1}}},
\end{aligned}
$$

where $\left|g_{l}\right| \leq 1$ and $\lim _{a \rightarrow 0} A_{l}=-1$. Using Lemma 3.3 once again, we can show that, for any subinterval $J \subset[0,1]$, we have

$$
\lim _{a \rightarrow 0} \frac{1}{a} \int_{J}\left(f_{h}-f_{l}\right) d \lambda=0 .
$$

For $J=[0,1]$ this means that the normalizations of $f_{l}$ and $f_{h}$ are asymptotically the same. Thus, the limit for a general $J$ implies that the $*$-weak limit of normalized measures defined using $f_{l}$ is the same as for those defined using $f_{h}$. Together with inequality (10) this proves Theorem 2.1.

\section{Computational Results}

We present in Fig. 2 graphs of $W_{a}$ normalized invariant densities for a): $a=0.1$, b): $a=0.05$ and c): $a=0.01$. They were obtained using Maple 13. Note that the vertical scales of the graphs are very different.

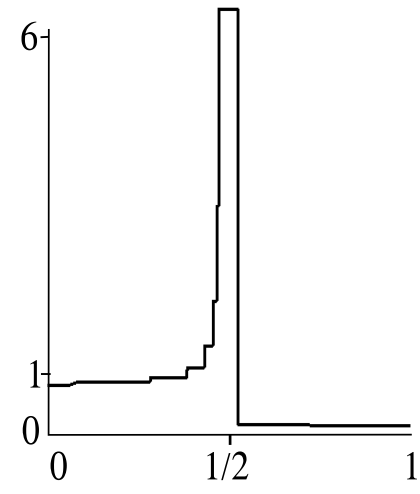

a)

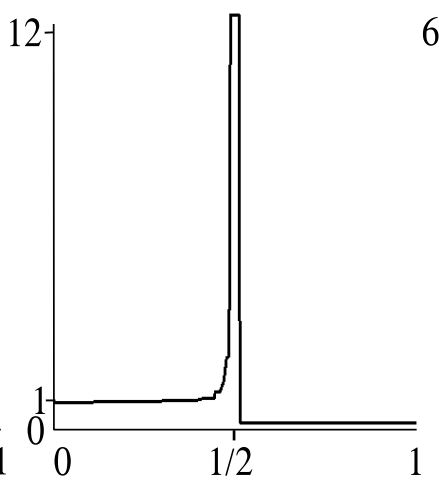

b)

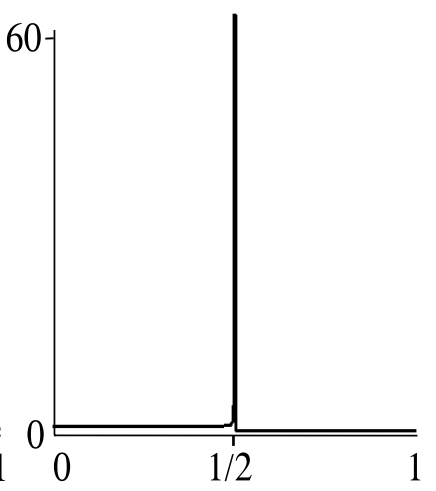

c)

Figure 2. $W_{a}$-invariant pdf's for a): $a=0.1, \mathrm{~b}$ ) $: a=0.05$ and c) $: a=0.01$.

Acknowledgment: The authors are grateful to an anonymous reviewer for comments.

Prepared using etds.cls 


\section{REFERENCES}

[1] V. Baladi and D. Smania, Alternative proofs of linear response for piecewise expanding unimodal maps, Ergodic Th. Dynamical Systems 30 (2010), 1-20, doi:10.1017/S0143385708001077.

[2] A. Boyarsky and P. Góra, Laws of Chaos. Invariant Measures and Dynamical Systems in One Dimension, Probability and its Applications, Birkhäuser, Boston, MA, 1997.

[3] M. Dellnitz, G. Froyland, and S. Seertl, On the isolated spectrum of the Perron-Frobenius operator, Nonlinearaity 13 (2000) 1171-1188.

[4] Dunford, N., and Schwartz, J.T, Linear Operators, Part I: General Theory, Interscience Publ. Inc., N.Y., 1964.

[5] P. Eslami and M. Misiurewicz, Singular limits of absolutely continuous invariant measures for families of transitive maps, J. Difference Equ. Appl., doi:10.1080/10236198.2011.590480.

[6] P. Góra, Invariant densities for piecewise linear maps of interval, Ergodic Th. and Dynamical Systems 29, Issue 05 (October 2009), 1549-1583.

[7] P. Góra, On small stochastic perturbations of one-sided subshift of finite type, Bull. Acad. Polon. Sci. 27 (1979), 47-51 .

[8] P. Góra and A. Boyarsky, Absolutely continuous invariant measures for piecewise expanding $C^{2}$ transformations in $R^{N}$, Israel Jour. Math. 67 (1989), 272-286.

[9] G. Keller, Stochastic stability in some chaotic dynamical systems, Monatshefte für Mathematik 94 (4) (1982) 313-333.

[10] G. Keller and C. Liverani., Stability of the spectrum for transfer operators, Ann. Scuola Norm. Sup. Pisa Cl. Sci. (4), 28 (1)(1999), 141-152.

[11] Z. S. Kowalski, Invariant measures for piecewise monotonic transformation has a positive lower bound on its support, Bull. Acad. Polon. Sci., Series des sciences mathematiques, 27, No. 1 (1979), 53-57.

[12] T.-Y. Li and J. A. Yorke, Ergodic transformations from an interval into itself, Trans. Amer. Math. Soc. 235 (1978), 183-192.

[13] R. Murray, Approximation of invariant measures for a class of maps with indifferent fixed points, University of Waikato, Mathematics Research Report Series II No. 106. (2005), http://www.math.canterbury.ac.nz/ r.murray/files/ulamifpnum.pdf

[14] C. G. Tokman, B. R. Hunt and P. Wright, Approximating invariant densities of metastable systems, arXiv:0905.0223v1 [math.DS], Ergodic Theory and Dynamical Systems 31 (2011), pp. 1345-1361, doi:10.1017/S0143385710000337.

Prepared using etds.cls 